\title{
Role of DNA-mismatch repair in anti-neoplastic effects of butyrate
}

\author{
S. S. Dronamraju ${ }^{1}$, J. M. Coxhead ${ }^{1}$, S. B. Kelly ${ }^{2}$ and J. C. Mathers ${ }^{1}$ \\ ${ }^{1}$ Human Nutrition Research Centre, Newcastle University, Newcastle Upon Tyne NE2 4HH, UK and ${ }^{2}$ North Tyneside \\ General Hospital, North Shields NE29 8NH, UK
}

Colo-rectal cancer (CRC) is the second-most-common cause of cancer-related death in the Western world ${ }^{(1)}$. DNA-mismatch repair (MMR) genes regulate key cellular processes, including correction of DNA replication errors ${ }^{(2)}$. Impaired functioning of MMR has been implicated in the aetiology of hereditary non-polyposis colon cancer and in $\leq 15 \%$ of sporadic $\mathrm{CRC}^{(2)}$. The $\mathrm{C}_{4}$ fatty acid, butyric acid, which is produced by bacterial fermentation of resistant starches in the large bowel, has potent anti-neoplastic effects on colon cancer cells $^{(3)}$. Recent in vitro studies have indicated that MMR status may modulate the anti-neoplastic effects of butyrate ${ }^{3}$. The present study aimed to investigate the mechanisms underlying these differential effects of butyrate on colon cancer cells.

SW48 colon cancer cells, in which the MMR gene MLH1 is silenced by promoter hypermethylation, were treated with the demethylating agent 5-aza 2 'deoxycytidine to partially demethylate and reactivate the $M L H 1$ gene. The native SW48 cells and their demethylated counterparts were treated with butyrate ( $0-5 \mathrm{~mm}$ ) for $8 \mathrm{~d}$ and the effects on cell proliferation, $M L H 1$ gene promoter methylation (combined bisulfite restriction analysis assay) and expression of two butyrate-responsive genes, i.e. CDK4 and GADD45A, were assessed (real-time RT-PCR).

Butyrate $(0.5-5 \mathrm{~mm})$ suppressed proliferation $(P<0.001)$ and reduced $M L H 1$ promoter methylation $(P<0.05)$ in SW48 cells. However, in demethylated SW48 cells butyrate caused a small but significant increase in cell proliferation $(P<0.05 ;$ Fig. 1$)$ and promoter methylation $(P<0.05)$. CDK4 expression was higher $(P=0.02)$ in demethylated SW48 cells compared with native SW48 cells. There was little effect of butyrate on $C D K 4$ expression in SW48 cells, but this was reduced markedly in the demethylated cells $(P=0.025$ for cell line $\times$ butyrate interaction; Fig: 2). Further there was more than two fold up regulation of GADD45A expression following butyrate (1 mm) treatment in native SW48 cells as compared with demethylated SW48 cells in which $G A D D 45 A$ expression was down regulated $(P=0.045$ for cell line $\times$ butyrate interaction; Fig: 2 ).

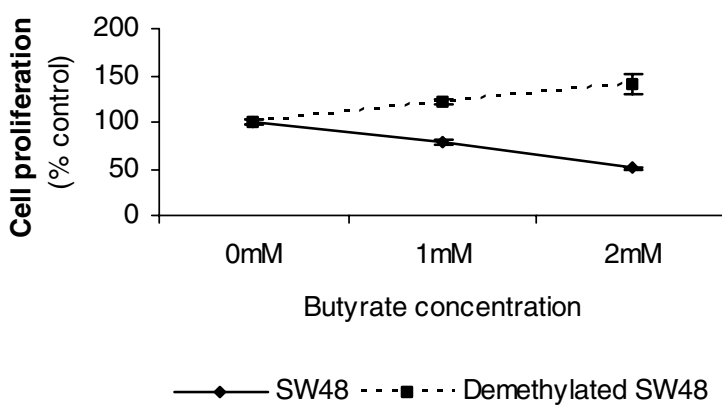

Fig. 1

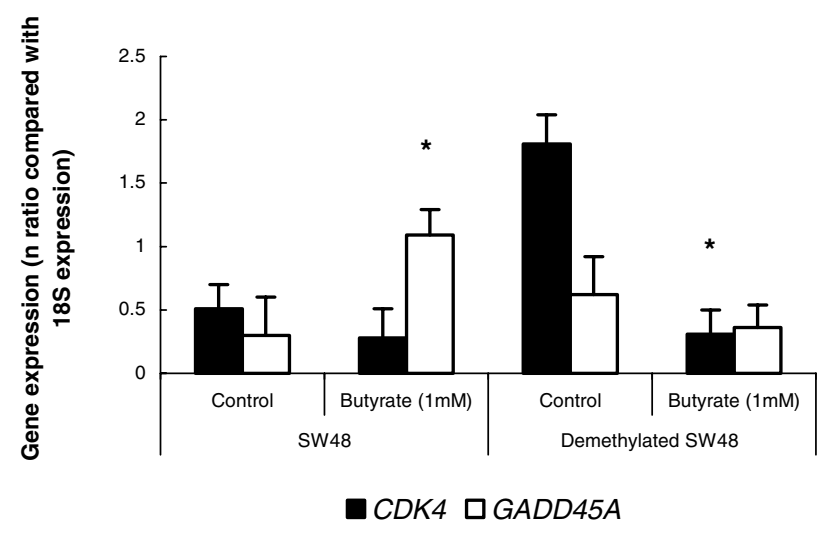

Fig. 2

The present study suggests that butyrate may have more potent anti-neoplastic effects on colon cancer cells, with epigenetic silencing of MLH1 function. Although butyrate showed differential expression of $C D K 4$ and GADD45A genes, it does not explain its effects on cell proliferation. It is essential to investigate the effects of butyrate on more cell-cycle regulatory genes to understand the molecular mechanisms underlying these differential effects.

This study was supported by the Biotechnology and Biological Sciences Research Council (grant no.13D/20173).

1. Cancer Research UK (2007) http://info.cancerresearchuk.org/cancerstats

2. Wheeler JM (2005) Ann R Coll Surg Engl 87, 15-20.

3. Coxhead JM, Williams EA \& Mathers JC (2005) Biochem Soc Trans 33, 728-729. 